\title{
Genetic Relationship of Body Energy and Blood Metabolites with Reproduction in Holstein Cows
}

\author{
G. Oikonomou, ${ }^{* 1}$ G. Arsenos, ${ }^{*}$ G. E. Valergakis, ${ }^{*}$ A. Tsiaras, $†$ D. Zygoyiannis, ${ }^{*}$ and G. Banos ${ }^{*}$ \\ *Department of Animal Production, Faculty of Veterinary Medicine, Box 393, Aristotle University of Thessaloniki, GR-54124 Thessaloniki, Greece \\ †Vivartia AVEE, Ziridi 10, Maroussi, GR-15123 Athens, Greece
}

\begin{abstract}
Body condition score (BCS), energy content (EC), cumulative effective energy balance (CEEB), and blood serum concentrations of glucose, B-hydroxybutyrate (BHBA), and nonesterified fatty acids (NEFA) were measured throughout first lactation in 497 Holstein cows raised on a large commercial farm in northern Greece. All these traits are considered to be indicators of a cow's energy balance. An additional measure of BCS, EC, and blood serum glucose, BHBA, and NEFA concentrations were taken approximately 2 mo (61 \pm $23 \mathrm{~d}$ ) before first calving. During first lactation, first service conception rate, conception rate in the first 305 $\mathrm{d}$ of lactation, interval from calving to conception, number of inseminations per conception, incidence of metritis, and incidence of reproductive problems of these cows were recorded; interval between first and second calving, and second lactation first service conception rate were also recorded. Random regression models were used to calculate weekly animal breeding values for first lactation BCS, EC, CEEB, glucose, BHBA, and NEFA. Single trait animal models were used to calculate breeding values for these traits measured on pregnant heifers before calving. Reproductive records were then regressed on animal breeding values for these energy balance-related traits to derive estimates of their genetic correlations. Several significant estimates were obtained. In general, traits that are known to be positively correlated with energy balance (BCS, EC, CEEB, and glucose) were found to have a favorable genetic relationship with reproduction, meaning that increased levels of the former will lead to an enhancement of the latter. On the other hand, traits known to be negatively correlated with energy balance (BHBA and NEFA) were found to have an unfavorable genetic association with reproductive traits. Body condition score, BHBA, and NEFA recorded early in lactation, and glucose concentrations measured in pregnant heif-
\end{abstract}

\footnotetext{
Received January 15, 2008.

Accepted June 27, 2008.

${ }^{1}$ Corresponding author: goikon@vet.auth.gr
}

ers had the highest genetic correlation with future reproductive performance. Results suggest that genetic selection for body energy and blood metabolites could facilitate the genetic improvement of fertility and overall reproductive efficiency of dairy cows.

Key words: genetic correlation, energy balance, metabolite, reproduction

\section{INTRODUCTION}

The continuing decline of fertility and reproductive efficiency in modern Holstein cows is undoubtedly one of the most important problems for the dairy industry to solve (Lucy, 2001; Pryce et al., 2004). Increased magnitude and duration of postpartum negative energy balance, arising from intense genetic selection for enhanced milk production, has been described as one of the main reasons responsible for this situation (Butler and Smith, 1989; de Vries and Veerkamp, 2000). Therefore, monitoring energy balance on a routine basis could facilitate improvement of fertility and reproduction.

The direct calculation of a cow's energy balance from estimates of feed intake and milk yield is quite difficult under field conditions, principally because of lack of feed intake records. Hence, the use of various energy balance indicators has been proposed for the study of the negative energy balance period and its relationship with reproduction. Body condition scoring is a subjective but reliable and widely accepted way of assessing a cow's body energy reserves (Edmonson et al., 1989; Fox et al., 1999). Body condition score and BW records can be used for the calculation of total body energy content (EC) and cumulative effective energy balance (CEEB), which have also been proposed for energy balance studies (Banos et al., 2006). Furthermore, various blood metabolites such as blood serum concentrations of glucose, BHBA, and NEFA have been reported to be strongly correlated to energy balance (Reist et al., 2002; Clark et al., 2005).

The relationship of some of the above energy balance indicators with cow reproduction has been examined in several studies. Low BCS or severe BCS loss after calv- 
Table 1. Descriptive statistics of BCS, energy content (EC), cumulative effective energy balance (CEEB), and blood serum concentrations of glucose, BHBA, and NEFA measured throughout first lactation

\begin{tabular}{lccrrrr}
\hline Trait (units of measurement) & Records (n) & Cows (n) & Mean & SD & Minimum & Maximum \\
\hline BCS (1 to 5) & 8,094 & 497 & 2.47 & 0.44 & 1.25 & 5.00 \\
EC (MJ) & 8,087 & 497 & $4,464.64$ & 946.99 & $2,328.90$ & $9,928.17$ \\
CEEB (MJ) & 8,087 & 497 & -423.79 & 973.83 & $-3,690.27$ & $5,984.08$ \\
Glucose (mg/dL) & 6,015 & 365 & 74.32 & 19.67 & 12.00 & 190.00 \\
BHBA (mmol/L) & 6,015 & 365 & 0.79 & 0.28 & 0.19 & 4.42 \\
NEFA (mmol/L) & 6,015 & 365 & 0.32 & 0.30 & 0.02 & 4.00 \\
\hline
\end{tabular}

ing was found to have adverse effects on cow reproductive performance (Suriyasathaporn et al., 1998; Pryce et al., 2001; Patton et al., 2007). Westwood et al. (2002) reported a significant positive phenotypic association of the ratio of glucose to BHBA during the first $10 \mathrm{wk}$ of lactation, with the probability of estrus expression at first ovulation. Furthermore, greater NEFA concentrations during the same time period were associated with lower probability of conception by d 150 of lactation (Westwood et al., 2002). Negative effects of high postpartum BHBA or NEFA blood concentrations on reproduction were also reported by Reist et al. (2000), Walsh et al. (2007), and Wathes et al. (2007).

Generally, most of the research on the relationship between energy balance and reproduction, including publications mentioned above, has been based on phenotypic studies. Nevertheless, evidence exists that part of this relationship is genetic (Veerkamp et al., 2000). Thus, an improvement of cow fertility and reproductive performance could potentially be achieved through genetic selection for energy balance-related traits. Before this becomes possible, however, the genetic correlation between such traits and reproduction must be established. Genetic correlation estimates of BCS, EC, and CEEB with reproductive traits have been reported in the past (Veerkamp et al., 2001; Banos et al., 2004; Wall et al., 2007). On the other hand, the genetic correlation of blood serum concentrations of glucose, BHBA, and NEFA, measured throughout or for a part of lactation, with reproductive traits has not been reported. Furthermore, the genetic correlation of all these traits measured in heifers before first calving with reproductive performance in first or subsequent lactations has not been established either.
The primary objective of this study was to investigate the genetic correlation of BCS, EC, CEEB, and serum concentrations of glucose, BHBA, and NEFA, measured throughout first lactation, with first and second lactation reproductive traits of Holstein cows. The genetic correlation of these traits measured on pregnant heifers before calving with first and second lactation reproductive performance was also studied.

\section{MATERIALS AND METHODS}

This research was conducted under the authority of the Faculty of Veterinary Medicine, Aristotle University of Thessaloniki. A protocol approved by the Faculty of Veterinary Medicine and the Research Committee of the Aristotle University of Thessaloniki was followed throughout the study.

\section{Population Description}

The study was conducted on a large commercial dairy farm in northern Greece $\left(41^{\circ} 2^{\prime} \mathrm{N}, 25^{\circ} 15^{\prime} \mathrm{E}\right.$, altitude $20 \mathrm{~m}$ ). Cows were housed in 4 free-stall barns and fed twice daily a TMR to meet their energy and protein requirements. Ration formulation was based on US National Research Council recommendations (NRC, 2001). Four hundred ninety-seven primiparous Holstein cows that calved, at an average age of 27.6 \pm 2.6 mo between January 2005 and July 2006, were included in the study. These animals had either been born on the farm or imported as pregnant heifers from 3 other European countries (Austria, France, and the Netherlands). The latter is a rather common practice among many commercial dairy farms in Greece.

Table 2. Descriptive statistics of BCS, energy content (EC), and blood serum concentrations of glucose, BHBA, and NEFA measured on heifers before calving

\begin{tabular}{lcrrrr}
\hline Trait (units of measurement) & Heifers (n) & \multicolumn{1}{c}{ Mean } & SD & Minimum & Maximum \\
\hline BCS (1 to 5) & 192 & 3.24 & 0.47 & 2.25 & 5.00 \\
EC (MJ) & 143 & $5,559.21$ & 951.22 & $3,751.59$ & $7,719.63$ \\
Glucose (mg/dL) & 174 & 70.60 & 19.80 & 20.00 & 122.00 \\
BHBA (mmol/L) & 175 & 0.55 & 0.19 & 0.24 & 1.57 \\
NEFA (mmol/L) & 142 & 0.49 & 0.41 & 0.05 & 2.50 \\
\hline
\end{tabular}


Table 3. Descriptive statistics of number of inseminations per conception (NINS) in first lactation, number of inseminations per conception for cows that conceived in the first $305 \mathrm{~d}$ of first lactation (NINS_305), interval from calving to conception for cows that conceived in the first $305 \mathrm{~d}$ of first lactation (CAL_CONC_305), interval between first and second calving (CI), first-lactation first-service conception rate (CONC_1AI1), conception rate in the first $305 \mathrm{~d}$ of first lactation (CONC_305), presence of metritis (METR) in first lactation, presence of reproductive problems (REPRO_PROB) in first lactation, and second-lactation firstservice conception rate (CONC_1AI2)

\begin{tabular}{lcrrrr}
\hline Trait (units of measurement) & Cows (n) & Mean & SD & Minimum & Maximum \\
\hline NINS & 315 & 2.97 & 1.82 & 1 & 11 \\
NINS_305 & 233 & 2.27 & 1.15 & 1 & 7 \\
CAL_CONC_305 (d) & 233 & 179.16 & 72.49 & 28 & 305 \\
CI (d) & 247 & 498.04 & 111.00 & 308 & 897 \\
CONC_1AI1 (0/1) & 375 & 0.19 & & & \\
CONC_305 (0/1) & 339 & 0.66 & & & \\
METR (0/1) & 497 & 0.09 & & & \\
REPRO_PROB (0/1) & 359 & 0.41 & & & \\
CONC_1AI2 (0/1) & 109 & 0.29 & & & \\
\hline
\end{tabular}

Cows were milked twice daily and their milk production was automatically recorded. Daily milk records were used for the calculation of 305-d yields.

Pedigree information was available for all cows in the herd. After considering all animals related to the cows of the present study, the total population size increased to 3,306, spanning the last 3 generations. All these data were included in the subsequent analyses.

\section{Body Energy and Blood Metabolites}

Cow BCS was assessed, after the morning milking, by a trained veterinarian on a weekly basis from calving to wk 13 of lactation and thereafter monthly, until the end of a 305-d lactation. A 5-point scale ( $1=$ emaciated, 5 = obese, scored in 0.25-point intervals) and the method described by Ferguson et al. (1994) were used. At the same time, the cows' BW was estimated using a heart girth tape (Webo, Aarup, Denmark), and blood was drawn from the coccygeal vein in a randomly selected subset (365 animals) of the studied cows.

Blood samples were left to clot at room temperature for approximately $30 \mathrm{~min}$ and then centrifuged at 2,000 $\times g$. The obtained serum samples were stored at $-20^{\circ} \mathrm{C}$ until analyzed for glucose, BHBA, and NEFA concentration. Serum glucose and NEFA were assayed colorimetrically using commercial kits (Glucose GOD-PAP method, P. Zafiropoulos S. A., Attiki, Greece and Wako NEFA C kit, Wako Chemicals GmbH, Neuss, Germany, respectively). The serum concentration of BHBA was assayed with the use of an enzymatic kinetic method based on the oxidation of B-hydroxybutyrate to acetoacetate by B-hydroxybutyrate dehydrogenase (Bruss, 1997). The intraassay coefficient of variation was 1 to $3 \%$ for glucose and 1.5 to $3 \%$ for NEFA, whereas the interassay coefficient was 3 to $5 \%$ for both glucose and NEFA. For BHBA, the intra- and interassay coefficients were 2 to $4 \%$ and 4 to $8 \%$, respectively.
The final data set consisted of 8,094 BCS, 8,087 estimated BW, and 6,015 serum glucose, BHBA, and NEFA concentration records. Body condition score and estimated BW records were used for the calculation of total body EC and CEEB with a procedure, which is described in detail by Banos et al. (2006). Briefly, empty body weight and total lipid and protein weights were predicted using the method of the US National Research Council (NRC, 2001) and were then combined to calculate EC. The latter was an estimate of the total energy in a cow's body at any given time of lactation. Changes in predicted lipid and protein weights from one week of lactation to the next were converted to effective energy as described by Banos et al. (2006), yielding estimates of CEEB. The latter represented body energy changes as accumulated throughout lactation. Table 1 shows descriptive statistics of all these traits.

Single measurements of BCS, EC, and serum concentrations of glucose, BHBA, and NEFA, taken approximately 2 mo $(61 \pm 23 \mathrm{~d})$ before calving, were also available for a subgroup of the studied animals. Cumulative effective energy balance was not possible to calculate here because it relates to energy changes between consecutive measurements and there was only a single record for each pregnant heifer. Descriptive statistics of these traits are in Table 2.

\section{Reproductive Traits}

Cows were observed for signs of estrus twice daily for $30 \mathrm{~min}$, as well as during the morning and afternoon milking. Cows detected in estrus were artificially inseminated by 2 experienced veterinarians $12 \mathrm{~h}$ later. Cows that did not exhibit estrus within 60 to $80 \mathrm{~d}$ postpartum joined a combined gonadotropin releasing hormone-prostaglandin estrus synchronization program, following the GPG protocol described by Stevenson et al. (1996). Pregnancy was diagnosed by rectal palpa- 
tion 40 to $55 \mathrm{~d}$ after insemination. Cows with signs of metritis were diagnosed by the farm's veterinarians and treated as appropriate.

All events regarding cow reproductive performance were systematically recorded. The following traits were derived from these records, spanning the time period from January 2005 to November 2007; all traits refer to first lactation unless otherwise stated: first-service conception rate $(0 / 1$; CONC_1AI1), conception rate (0/1) in the first $305 \mathrm{~d}$ of lactation (CONC_305), number of inseminations per conception (NINS), number of inseminations per conception for cows that conceived in the first $305 \mathrm{~d}$ of lactation (NINS_305), interval (d) from calving to conception for cows that conceived in the first $305 \mathrm{~d}$ of lactation (CAL_CONC_305), presence (0/1) of metritis (METR), presence (0/1) of reproductive problems (REPRO_PROB) defined as diagnosed metritis or failure to conceive within the first $305 \mathrm{~d}$ of lactation, interval (d) between the cow's first and second calving (CI), and second-lactation first service conception rate $\left(0 / 1 ; \mathbf{C O N C} \_1 \mathrm{AI} 2\right)$. In this study, the trait REPRO_PROB was defined as an index of different reproductive problems, namely presence of metritis and failure to conceive, with practical interest to the farmer. Descriptive statistics for these traits are presented in Table 3. Information on some traits was not available for animals that were culled. Because the main reason that led to involuntary culling was lameness, all lameness incidences were recorded and included in the analysis.

\section{Statistical Analysis}

Weekly breeding values of all animals for BCS, EC, CEEB, glucose, BHBA, and NEFA were calculated with the following random regression model; each trait was analyzed separately:

$$
\begin{aligned}
& Y_{i j k m n}=Y S_{i}+C_{j}+a_{1} \cdot \text { age }+\sum_{n=0}^{3} b_{n} P_{n} W_{m} \\
& +\sum_{n=0}^{3} c_{k n} P_{n} W_{m}+\sum_{n=0}^{1} d_{k n} P_{n} W_{m}+e_{i j k m n},
\end{aligned}
$$

where $Y_{i j k m n}=$ record of cow $k$ in week of lactation $m$, $\mathrm{YS}_{\mathrm{i}}=$ fixed effect of year-season of calving $i$ (4 levels), $C_{j}$ $=$ fixed effect of country of origin $j$ (4 levels), $a_{1}=$ linear regression coefficients on age at calving (age), $W_{m}=$ week of lactation $m$ (44 levels), $b_{n}=$ fixed regression coefficient on week of lactation, $c_{k n}=$ random regression coefficient on week of lactation associated with the genetic effect of cow $k$ including the full animal pedigree relationship matrix, $d_{k n}=$ random regression coefficient on week of lactation associated with the permanent environmental effect of cow $k, P_{n}=n$th orthogonal polynomial of week $m(n+1=$ order of polynomial), and $e_{i j k m n}=$ random residual term ( 4 classes as described below).

In model 1, the fixed regression coefficient was associated with an overall, average lactation curve for each trait, whereas the random regression was associated with each individual cow's deviation from the overall curve. Breeding values were estimated for all animals, during each week of lactation, by combining solutions for the fixed curve and random deviation.

The full model 1 was applied to the analysis of BCS, EC, and CEEB. For the analysis of blood serum glucose, BHBA, and NEFA concentrations, the effects of country of origin and age at calving were not included because preliminary analyses showed that they were not significantly different from zero $(P>0.05)$.

Fourth-order orthogonal polynomial was chosen following preliminary analyses of different (lower) orders and comparisons between models using the log-likelihood, until the latter stopped changing significantly ( $P$ $>0.05$ ) with increasing polynomial order. This was true for all traits except BHBA where third order was the maximum significant.

Permanent environment was modeled with a secondorder orthogonal polynomial. Efforts to increase the order were unsuccessful as they led to convergence problems. This may be a function of the data size, although the number of observations is not deemed too small for a controlled study. Another explanation could be that higher order regression might have actually over-fit first-lactation permanent environmental effects. For the analysis of CEEB, glucose, and NEFA, a first-order permanent environmental polynomial was the most highly significant.

Depending on lactation stage, 4 measurement error classes were defined as follows: wk 1 to 4,5 to 9 , 10 to 21 , and $>21$. Different residual variances were estimated for each measurement error class, whereas covariances between classes were zero.

Single records, taken on pregnant heifers before calving, were analyzed with a model similar to model 1 that included the effect of days to calving but excluded the fixed and random regressions on week of lactation. Animal solutions derived from this model represented breeding values for the studied traits.

Model 2 was used to determine the genetic correlation of body energy (BCS, EC, CEEB) and blood metabolite (glucose, BHBA, NEFA) traits with reproductive performance (NINS, NINS_305, CAL_CONC_305, CI, CONC_1AI1, CONC_305, METR, REPRO_PROB, CONC_1AI2); each reproductive trait was analyzed separately. 


$$
\begin{gathered}
Y_{i j k l m}=Y S_{i}+G_{j}+S_{k}+L_{l}+a_{1} \cdot \text { age }+ \\
a_{2} \cdot \operatorname{dim}+a_{3} \cdot \text { milk }+a_{4} \cdot \text { proof } \\
+ \text { cow }_{m}+e_{i j k l m},
\end{gathered}
$$

where $Y_{i j k l m}=$ reproductive record of cow $m, Y S_{i}=$ fixed effect of year-season of calving $i, G_{j}=$ fixed effect indicating whether the cow was included in an estrus synchronization scheme $(j=0,1), S_{k}=$ fixed effect of season of first $\mathrm{AI} k, L_{l}=$ fixed effect indicating whether the cow was diagnosed with lameness at any time throughout lactation $(l=0,1), a_{1}=$ linear regression coefficients on age at calving (age), $a_{2}=$ linear regression coefficients on days in milk at first AI (dim), $a_{3}=$ linear regression coefficients on 305-d milk yield (milk), $a_{4}=$ linear regression coefficients on animal breeding value for body energy or blood metabolite (proof), cow $_{m}=$ random effect of cow $m$ (including all known pedigree), and $e_{i j k l m}$ = random residual term.

Following preliminary analyses, only fixed effects with a significant $(P<0.05)$ effect on a reproductive trait were included in each analysis. Thus, the estrus synchronization effect $(G)$ was included in the analysis of conception rate traits only; season of first insemination (S) was included in the analysis of conception rate after first insemination (first and second lactation), number of inseminations per conception, and interval from calving to conception; and lameness (L) was included in the analysis of calving interval, reproductive problems, and conception rate in the first $305 \mathrm{~d}$ of lactation. All other effects shown in model 2 were included in all analyses. The effect of 305-d milk yield was included to derive solutions for constant production level.

In model 2, the regression of interest was that on animal breeding value for BCS, EC, CEEB, glucose, BHBA, and NEFA. The latter were weekly breeding values derived from model 1 or single-trait breeding values for pregnant heifer traits. Each breeding value for each trait was fitted separately in a series of con- secutive analyses. In this context, regression of a reproductive record on the breeding value for a body energy or blood metabolite represented a genetic prediction of the former from the latter. Predictions of first-lactation reproductive traits were derived from weekly genetic evaluations for BCS, EC, CEEB, glucose, BHBA, and NEFA, measured during the first 13 wk of the first lactation, which represents the period before the onset of reproductive activity. Predictions of CONC_1AI2 were derived from genetic evaluations for the same body energy and blood metabolites, obtained during the entire first lactation. Predictions of all traits were derived from breeding values for BCS, EC, glucose, BHBA, and NEFA measured on pregnant heifers before first calving.

All analyses were performed with the use of the ASREML software package (Gilmour et al., 2002). Pairwise regressions of reproductive traits on genetic evaluations for BCS, EC, CEEB, glucose, BHBA, and NEFA were used to estimate approximate genetic correlations between these groups of traits, with the following equation:

$$
r_{G}=b \cdot \frac{\sigma_{1}}{\sigma_{2}}
$$

where $r_{G}=$ the estimated genetic correlation between 2 traits, $b=$ the regression coefficient calculated from model 2 , and $\sigma_{1}$ and $\sigma_{2}=$ the genetic standard deviation estimates for the body energy or metabolite and the reproductive trait, respectively. The REML estimates of genetic standard deviations for BCS, EC, CEEB, glucose, BHBA, and NEFA in each week of lactation were obtained with model 1. Genetic standard deviation estimates for reproductive traits were obtained with a model similar to model 2 without the regressions on body energy or metabolite genetic evaluation and 305d milk yield.

Approximate standard errors of estimated genetic correlations were based on the following equation:

Table 4. Maximum genetic correlation estimates (SE) of weekly BCS and energy content (EC) with interval between first and second calving (CI), first-lactation first-service conception rate (CONC_1AI1), presence of metritis (METR) in first lactation, and presence of reproductive problems (REPRO_PROB) in first lactation

\begin{tabular}{lccccccc}
\hline & \multicolumn{3}{c}{ BCS } & & \multicolumn{3}{c}{ EC } \\
\cline { 2 - 3 } Trait & $\begin{array}{r}\text { Week of } \\
\text { lactation }\end{array}$ & Estimate & $P$-value & & $\begin{array}{c}\text { Week of } \\
\text { lactation }\end{array}$ & Estimate & $P$-value \\
\hline CI & 1 & $-0.27(0.16)$ & 0.09 & & & & \\
CONC_1AI1 & 12 & $0.22(0.11)$ & 0.05 & & & & \\
METR & 2 & $-0.28(0.14)$ & 0.05 & & 6 & $-0.30(0.15)$ & 0.05 \\
REPRO_PROB & 5 & $-0.23(0.11)$ & 0.04 & & 2 & $-0.20(0.10)$ & 0.03 \\
\hline
\end{tabular}




$$
V\left(r_{G}\right) \cong r_{G}^{2} \cdot\left[\frac{V(b)}{b^{2}}+\frac{V\left(\sigma_{1}^{2}\right)}{4 \cdot \sigma_{1}^{4}}+\frac{V\left(\sigma_{2}^{2}\right)}{4 \cdot \sigma_{2}^{4}}\right],
$$

where $V\left(r_{G}, b, \sigma_{1}\right.$, or $\left.\sigma_{2}\right)=$ the standard error of each estimate squared; other effects are as defined in equation [3].

All genetic correlation estimates in this study were derived from regressions of reproductive traits on breeding values for body energy and blood metabolites. Similar approaches to estimating genetic correlations have been previously reported (Brotherstone and Hill, 1991; Pryce et al., 2000; Banos et al., 2006). A more direct method would be to calculate parameters with a bivariate REML analysis. However, we could not manage to combine a trait with repeated records (body energy, blood metabolites) with a single observation trait (reproduction) in such an analysis. Although it was possible to theoretically build the necessary (co) variance matrix, it was not practically and computationally feasible to calculate estimates. Alternatively, estimates of genetic correlations could be also derived from bivariate analyses of single weekly body energy and blood metabolic records with reproduction traits. This method, although feasible, would treat repeated measures of an animal as independent records. Therefore, we opted for the approach described above, which recognizes covariances among repeated measures and properly describes their trajectory. For purposes of comparison, a bivariate analysis of single weekly BCS records with reproductive traits was also performed. In addition to producing comparative results, this would provide a measure of the level of residual (environmental) covariance between traits that might have affected our chosen method.

\section{RESULTS}

The present study revealed several genetic correlation estimates between traits that were statistically significant $(P<0.05)$ or tended to be significant $(P<0.10)$.
Maximum genetic correlation estimates of weekly firstlactation BCS and EC with CI, CONC_1AI1, METR, and REPRO_PROB are presented in Table 4. Maximum genetic correlation estimates of weekly first-lactation BHBA and NEFA with NINS, CAL_CONC_305, CI, CONC_1AI1, METR, and REPRO_PROB are presented in Table 5.

Tables 4 and 5 include only pairs of traits with genetic correlation estimates that were statistically significant $(P<0.05)$ or tended to be significant $(P<0.10)$. Other weekly estimates were also significant. Thus, genetic correlation of BCS with CI ranged from -0.26 to -0.27 (wk 1 to $2, P<0.10$ ), with METR ranged from -0.24 to $-0.27(P<0.05$ for wk 1 to 2 and $P<0.10$ for wk 3$)$, with REPRO_PROB ranged from -0.21 to $-0.23(P<$ 0.05 for wk 1 to 7 and $P<0.10$ for wk 8 to 9), and with CONC_1AI1 ranged from 0.23 to $0.26(P<0.05$ for wk 12 to 13 and $P<0.10$ for wk 10 to 11 ). Furthermore, genetic correlation of EC with METR ranged from -0.28 to -0.30 ( $P<0.05$ for wk 1 to 4$)$, and REPRO_PROB ranged from -0.19 to $-0.20(P<0.05$ for wk 1 to 3 and $P<0.10$ for wk 4 to 5 ).

Genetic correlation of blood serum BHBA concentrations with NINS ranged from 0.55 to $0.56(P<0.05$ for wk 1 to 7 and $P<0.10$ for wk 8), with CI ranged from 0.34 to $0.41(P<0.05$ for wk 6 to 7 and $P<0.10$ for wk 1 to 5 and wk 8), with REPRO_PROB ranged from 0.31 to $0.34(P<0.05$ for wk 1 to 8 and $P<0.10$ for wk 9$)$, with CONC_1AI1 was $-0.22(P<0.05$ for wk 1 to 6 and $P<0.10$ for wk 7$)$, and with CONC_305 ranged from -0.65 to $-0.69(P<0.05$ for wk 1 to 8$)$.

The genetic correlation between blood serum NEFA concentrations and CI was 0.30 (wk 8 to $9, P<0.10$ ), whereas between NEFA concentrations and METR ranged from 0.31 to $0.42(P<0.05$ for wk 12 to 13 and $P<0.10$ for wk 11). Genetic correlation estimates of NEFA concentrations with REPRO_PROB ranged from 0.21 to 0.22 (wk 8 to $9, P<0.10$ ), with CONC_305 ranged from -0.27 to -0.29 (wk 1 to $5, P<0.10$ ), and with CONC_1AI1 was -0.17 (wk $1, P=0.10)$.

Table 5. Maximum genetic correlation estimates (SE) of weekly blood serum BHBA and NEFA with number of inseminations per conception (NINS) in first lactation, conception rate in the first $305 \mathrm{~d}$ of first lactation (CONC_305), interval between first and second calving (CI), first-lactation first-service conception rate (CONC_1AI1), presence of metritis (METR) in first lactation, and presence of reproductive problems (REPRO_PROB) in first lactation

\begin{tabular}{|c|c|c|c|c|c|c|}
\hline \multirow[b]{2}{*}{ Trait } & \multicolumn{3}{|c|}{ BHBA } & \multicolumn{3}{|c|}{ NEFA } \\
\hline & $\begin{array}{l}\text { Week of } \\
\text { lactation }\end{array}$ & Estimate & $P$-value & $\begin{array}{l}\text { Week of } \\
\text { lactation }\end{array}$ & Estimate & $P$-value \\
\hline NINS & 3 & $0.56(0.23)$ & 0.02 & & & \\
\hline CONC_305 & 1 & $-0.65(0.23)$ & 0.01 & & & \\
\hline CI & 6 & $0.41(0.21)$ & 0.05 & 8 & $0.30(0.18)$ & 0.10 \\
\hline CONC_1AI1 & 1 & $-0.22(0.11)$ & 0.04 & 1 & $-0.17(0.10)$ & 0.10 \\
\hline METR & & & & 13 & $0.42(0.20)$ & 0.03 \\
\hline REPRO_PROB & 1 & $0.34(0.13)$ & 0.01 & 8 & $0.22(0.12)$ & 0.07 \\
\hline
\end{tabular}


All other genetic correlation estimates between pairs of reproductive and first lactation body energy or blood metabolites were not significant. In some cases, however, the genetic regressions, from which genetic correlations were derived, were significantly different from zero $(P<0.05)$, without the latter being so. This was mainly because of large standard errors associated with reproductive trait genetic variance estimates. Nonsignificant correlations derived from statistically significant $(P<0.05)$ regressions were between wk 10 to $13 \mathrm{EC}$ and CAL_CONC_305 (-0.66 to -0.74 , $P=0.28$ to 0.30 ), wk 3 to 13 CEEB and NINS_305 ( -0.48 to $-0.75, P=0.35$ to 0.65 ), wk 5 to $13 \mathrm{CEEB}$ and CAL_CONC_305 ( -0.55 to $-0.65, P=0.27$ to 0.33$)$, wk 12 to 13 blood serum glucose concentrations and CAL_CONC_305 $(-0.52, P=0.30)$, and wk 5 blood serum glucose concentrations and CONC_1AI2 (0.39, $P$ $=0.11$ ).

Finally, the genetic correlation between blood serum glucose concentrations measured on pregnant heifers before the first calving was $-0.21(P=0.12)$ with CI and $-0.40(P=0.10)$ with REPRO_PROB. Genetic correlations of all the other body energy and blood metabolites, measured on pregnant heifers, with reproductive performance were not significantly different from zero $(P>0.05)$ and did not show any such tendency either $(P>0.10)$.

\section{DISCUSSION}

Genetic correlation estimates of BCS with reproductive traits have been previously reported in several studies. In all previous studies, a favorable genetic correlation between BCS and reproduction of Holstein cows was found, which is supported by results of this study. For example, Pryce et al. (2000) reported the genetic correlation between sire estimated breeding value for BCS and their daughters' CI to be -0.40 . After adjusting for phenotypic milk yield, to derive estimates for a constant production level, the genetic correlation was -0.22 , which is quite close to estimates of the present study. Furthermore, Berry et al. (2003) used random regression models to estimate the genetic correlation between BCS and fertility traits. The genetic correlation with pregnancy rate at first service reported in their study ( 0.15 to 0.38 ) was similar to the correlation between BCS and CONC_1AI1 estimated here (0.17 to 0.21$)$. Results from the present study, regarding genetic correlations between BCS and fertility traits, are also within the range of results reported by Dechow et al. (2001), Veerkamp et al. (2001), Banos et al. (2004), and Wall et al. (2007).

Wall et al. (2007) presented a significantly negative genetic correlation between EC and CI. In this study, this correlation was also negative, though not significant. Given the positive association between EC and energy balance, a negative (favorable) genetic correlation with CI is reasonable and indicative of the potential to decrease the interval between consecutive calvings by improving the body energy content of high producing cows.

Although phenotypic correlations of blood concentrations of glucose, BHBA, and NEFA with various reproductive and fertility traits have been reported in previous studies, genetic correlation estimates between these traits are largely missing from the available literature. Hayhurst et al. (2007) estimated a negative genetic correlation $(-0.44)$ between NEFA blood concentrations in Holstein male calves and their estimated breeding value for female fertility traits. This finding, though not directly comparable with ours, suggests that genetic predisposition for high NEFA concentrations may be associated with compromised reproductive performance. Positive genetic correlations of NEFA concentrations with calving interval, metritis, and reproductive problems, and a negative genetic correlation between NEFA and conception rate following first AI calculated in the present study support this assertion. In the study of Hayhurst et al. (2007), a negative (unfavorable) but nonsignificant genetic correlation between blood glucose concentrations in Holstein male calves and their estimated breeding value for female fertility traits was also reported. On the contrary, results of this study suggest that there may be a favorable genetic association between glucose concentrations measured in early lactation and reproduction. Although genetic correlation estimates were not statistically significant, the genetic regression from which these estimates were derived was significant. Thus, elevated glucose concentrations were linked to decreased interval from calving to conception and increased rate of conception following first insemination. Furthermore, we found glucose concentrations in heifers to be genetically related with a reduced interval between first and second calving and fewer reproductive problems in first lactation. Reist et al. (2002) reported that glucose concentrations have been found to be positively correlated with energy balance. There is also evidence that glucose is the main energy source for the ovary (Rabiee et al., 1999), whereas phenotypic studies have shown positive correlation between glucose concentrations and fertility (Moallem et al., 1997; Westwood et al., 2002). Thus, the tendency toward a favorable genetic relationship of blood glucose concentrations in heifers and cows with reproductive performance reported in the present study appears plausible. However, given the largely nonsignificant estimates obtained, there is need for further investigation of this correlation. 
Among the blood metabolites, NEFA concentration is assumed to be the best indicator of a cow's energy balance (Reist et al., 2002) because elevated NEFA concentration is the first indication of lipolysis. However, in our study it was BHBA concentrations that were found to have the strongest genetic correlation with reproductive traits. In the liver, NEFA can be esterified to triglycerides, enter the citric acid cycle, or form ketone bodies (Holtenius and Holtenius, 1996). High rates of ketogenesis and high blood BHBA concentrations can be considered as cow's failure to adapt to negative energy balance (Herdt, 2000). It is possible that cows genetically prone to lower reproductive ability are those cows that are also prone to not only mobilize greater amounts of their energy reserves but also turn a greater proportion of NEFA released from this mobilization to ketone bodies (i.e., BHBA). In other words, there might be a genetic background in the ability of dairy cows to regulate NEFA concentrations and hence to adapt to negative energy balance; this genetic variation could explain part of the genetic relationship between energy balance and fertility.

Results from the present study support the idea that there is a substantial genetic background in the relationship between energy balance and reproduction in dairy cows. Traits studied here that are positively correlated to energy balance (BCS, EC, CEEB, and glucose) were found to have a generally favorable genetic correlation with reproductive traits. On the contrary, traits known to be negatively correlated with energy balance (BHBA and NEFA) were found to have an unfavorable genetic correlation with reproduction; the latter being characterized by conception (CONC_1AI1, CONC_305, CONC_1AI2, NINS, NINS_305) as well as interval (CI, CAL_CONC_305) traits. Admittedly, reproduction is a rather complicated process related to the cow's ability to exhibit estrus, conceive, and carry the pregnancy to term. In this context, the array of reproductive traits studied here effectively covers most of the process. The fact that cows, which did not exhibit estrus in the first 3 mo of lactation, underwent hormonal treatment to elicit and synchronize estrus may mask the assessment of their natural reproductive behavior. However, this is a usual practice in large commercial farms. Furthermore, the effect was recorded and accounted for in the analysis model (model 2); therefore, it is not expected to have influenced our results.

Regarding the stage of lactation in which significant correlations were observed, we note that most of these correlations were found in the first weeks of lactation. This is quite reasonable because this is the period in which the cow goes through negative energy balance, and therefore, this is the period in which energy bal- ance indicators are expected to be more informative. Significant genetic correlations between BCS and CONC_1AI1 were observed during the 12th and 13th week of lactation. This is the period where most of the cows were inseminated for the first time, and it may explain the significance of the specific correlations during the period.

Significant genetic correlations between BCS, EC, or NEFA concentrations and metritis reported in this study suggest that energy balance is genetically associated not only with dairy cow reproductive ability but with their reproductive health, too. Phenotypic associations of energy balance traits, especially BCS, with dairy cow reproductive health have already been reported (Markusfeld et al., 1997). Apparently, part of this association, as far as metritis is concerned, is because of genetic factors, and it is possible that genetic selection of cows for energy balance traits (e.g., BCS) will enhance their genetic resistance to metritis. The possible existence of similar genetic associations of energy balance traits with other health disorders could be the objective of future studies.

The methodology used here for the estimation of genetic correlations is an approximation based on the assumption of zero residual covariance between traits. The method has been shown to work well when sire breeding values for one trait were combined with daughter records for the other (Brotherstone and Hill, 1991; Pryce et al., 2000). Furthermore, Banos et al. (2006) used the exact same approach with cow breeding values and a data set similar to ours. In the present study, we ran an additional series of bivariate analyses of weekly BCS and reproduction to compare results and also assess the size of the residual covariance between traits; such analyses were conducted sequentially for each week of lactation (e.g., wk 1 BCS - reproductive trait, wk 2 BCS - reproductive trait, etc). Estimated genetic correlations were very consistent with results presented in this manuscript, at least with regard to sign and significance levels. In addition, residual correlations were practically zero. For example, genetic correlations between weekly BCS and CI ranged from -0.12 to -0.49 (SE 0.07 to 0.10 ); residual correlations between the same traits ranged from 0 to -0.07 (SE 0.07 to 0.08 ). It should be noted that these bivariate analyses treated repeated records of an animal as independent observations and did not account for the trajectory in the way random regressions did. For this reason, we opted for the analysis of breeding values derived from random regression models. Consistency of the genetic correlation estimates from the 2 methods and the negligible residual covariance estimates give confidence in the results. Furthermore, similarities 
of the results presented here with various literature estimates using different methods, as discussed above, add further confidence to our chosen approach.

In a separate series of analyses, the regression of reproductive traits on breeding values was weighed by the latter's reliability to account for incomplete information. However, results were not affected.

Previous studies have already proposed the incorporation of BCS records in fertility traits selection indices and this is now happening in certain countries' breeding and selection schemes. Results presented here confirm the relevance and usefulness of BCS in the potential genetic improvement of dairy cow fertility. Furthermore, results presented in this study show that there is an opportunity for genetic improvement of lowly heritable reproductive traits through selection for other body energy and blood metabolites. Concentrations of BHBA in blood serum, especially during the first weeks of lactation, seem to be the most interesting of these traits, as it was found to be strongly correlated with several reproductive traits. Moreover, BHBA concentrations measured during the first weeks of lactation could be used for management purposes. For example, the identification and treatment of cows suffering from subclinical ketosis can be facilitated by monitoring BHBA concentrations. Benefits accruing from routine BHBA concentration recording may justify the expenses required for such measurements, including costs for blood sampling and laboratory analyses, especially in high production or breeding herds. Furthermore, recording blood serum glucose and NEFA concentrations in the early stages of first lactation could also contribute to the genetic improvement of fertility and provide additional management tools (Whitaker, 2004).

\section{CONCLUSIONS}

Useful genetic correlations of body energy and blood metabolites measured throughout first lactation with cow reproductive performance, derived in this study, demonstrate the possibility to use records of the former in an effort to improve dairy cow fertility through genetic selection. Body condition score and blood serum BHBA and NEFA concentrations recorded early in lactation appear to be the most interesting traits. Parameters calculated in the present study can be used to develop enhanced fertility indices including reproductive as well as indicator traits.

\section{ACKNOWLEDGMENTS}

Funding for this research was made available from the General Secretariat for Research and Technology of the Greek Ministry of Development. The first author acknowledges financial support from the Greek State Scholarships Foundation.

\section{REFERENCES}

Banos, G., S. Brotherstone, and M. P. Coffey. 2004. Evaluation of body condition score measured throughout lactation as an indicator of fertility in dairy cows. J. Dairy Sci. 87:2669-2676.

Banos, G., M. P. Coffey, E. Wall, and S. Brotherstone. 2006. Genetic relationship between first lactation body energy and later life udder health in dairy cattle. J. Dairy Sci. 89:2222-2232.

Berry, D. P., F. Buckley, P. Dillon, R. D. Evans, M. Rath, and R. F. Veerkamp. 2003. Genetic parameters for body condition score, body weight, milk yield, and fertility estimated using random regression models. J. Dairy Sci. 86:3704-3717.

Brotherstone, S., and W. G. Hill. 1991. Dairy herd life in relation to linear type traits and production 1. Phenotypic and genetic analyses in pedigree type classified herds. Anim. Prod. 53:279287.

Bruss, M. L. 1997. Lipids and Ketones. Pages 83-115 in Clinical Biochemistry of Domestic Animals. 5th ed. J. J. Kaneko, J. W. Harvey, and M. L. Bruss, ed. Academic Press, San Diego, CA.

Butler, W. R., and R. D. Smith. 1989. Interrelationship between energy balance and postpartum reproductive function in dairy cattle. J. Dairy Sci. 72:767-778.

Clark, C. E. F., W. J. Fulkerson, K. S. Nandra, I. Barchia, and K. L. Macmillan. 2005. The use of indicators to assess the degree of mobilization of body reserves in dairy cows in early lactation on a pasture-based diet. Livest. Prod. Sci. 94:199-211.

de Vries, M. J., and R. F. Veerkamp. 2000. Energy balance of dairy cattle in relation to milk production variables and fertility. J. Dairy Sci. 83:62-69.

Dechow, C. D., G. W. Rogers, and J. S. Clay. 2001. Heritabilities and correlations among body condition scores, production traits and reproductive performance. J. Dairy Sci. 84:266-275.

Edmonson, A. J., I. J. Lean, L. D. Weaver, T. Farver, and G. Webster. 1989. A body condition scoring chart of Holstein dairy cows. J. Dairy Sci. 72:68-78.

Ferguson, J. D., D. T. Galligan, and N. Thomsen. 1994. Principal descriptors of body condition in Holstein dairy cattle. J. Dairy Sci. 77:2695-2703.

Fox, D. G., M. E. Van Amburgh, and T. P. Tylutki. 1999. Predicting requirements for growth, maturity and body reserves in dairy cattle. J. Dairy Sci. 82:1968-1977.

Gilmour, A. R., B. J. Gogel, B. R. Cullis, S. J. Welham, and R. Thompson. 2002. ASREML User's guide, Release 1.0. VSN International Ltd., Hemel Hempstead, UK.

Hayhurst, C., M. K. Sørensen, M. D. Royal, and P. Løvendahl. 2007. Metabolic regulation in Danish bull calves and the relationship to the fertility of their female offspring. J. Dairy Sci. 90:39093916.

Herdt, T. H. 2000. Ruminant adaptation to negative energy balance. Vet. Clin. North Am. 16:215-230.

Holtenius, P., and K. Holtenius. 1996. New aspects of ketone bodies in energy metabolism of dairy cows: A review. J. Vet. Med. A 43:579-587.

Lucy, M. C. 2001. Reproductive loss in high-producing dairy cattle: Where will it end? J. Dairy Sci. 84:1277-1293.

Markusfeld, O., N. Gallon, and E. Ezra. 1997. Body condition score, health, yield and fertility in dairy cows. Vet. Rec. 141:67-72.

Moallem, U., M. Kaim, Y. Folman, and D. Sklan. 1997. Effect of calcium soaps of fatty acids and administration of somatotropin in early lactation on productive and reproductive performance of high producing dairy cows. J. Dairy Sci. 80:2127-2136.

NRC. 2001. Nutrient Requirements of Dairy Cattle. 7th ed. Natl. Acad. Press, Washington, DC.

Patton, J., D. A. Kenny, S. McNamara, J. F. Mee, F. P. O’Mara, M. G. Diskin, and J. J. Murphy. 2007. Relationships among milk production, energy balance, plasma analytes, and reproduction in Holstein-Friesian cows. J. Dairy Sci. 90:649-658. 
Pryce, J. E., M. P. Coffey, and S. Brotherstone. 2000. The genetic relationship between calving interval, condition score and linear type and management traits in pedigree registered Holstein dairy cows. J. Dairy Sci. 83:2664-2671.

Pryce, J. E., M. P. Coffey, and G. Simm. 2001. The relationship between body condition score and reproductive performance. J. Dairy Sci. 84:1508-1515.

Pryce, J. E., M. D. Royal, P. C. Garnsworthy, and I. L. Mao. 2004. Fertility in the high-producing dairy cow. Livest. Prod. Sci. 86:125-135.

Rabiee, A. R., I. J. Lean, J. M. Gooden, and B. G. Miller. 1999 Relationships among metabolites influencing ovarian function in the dairy cow. J. Dairy Sci. 82:39-44.

Reist, M., D. Erdin, D. von Euw, K. Tschuemperlin, H. Leuenberger, Y. Chilliard, M. Hammon, C. Morel, C. Philipona, Y. Zbinden, N Kuenzi, and J. W. Blum. 2002. Estimation of energy balance at the individual and herd level using blood and milk traits in high yielding cows. J. Dairy Sci. 85:3314-3327.

Reist, M., A. Koller, A. Busato, U. Kupfer, and J. W. Blum. 2000. First ovulation and ketone body status in the early postpartum period of dairy cows. Theriogenology 54:685-701.

Stevenson, J. S., Y. Kobayashi, M. P. Shipka, and K. C. Rauchholz 1996. Altering conception of dairy cattle by gonadotropinreleasing hormone preceding luteolysis induced by prostaglandin F2a. J. Dairy Sci. 79:402-410.

Suriyasathaporn, W., M. Nielen, S. J. Dieleman, A. Brand, E. N. Noordhuizen-Stassen, and Y. H. Schukken. 1998. A Cox proportional-hazards model with time-dependent covariates to evaluate the relationship between body condition score and the risks of first insemination and pregnancy in a high producing dairy herd. Prev. Vet. Med. 37:159-172.

Veerkamp, R. F., E. P. C. Koenen, and G. De Jong. 2001. Genetic correlations among body condition score, yield, and fertility in first-parity cows estimated by random regression models. J. Dairy Sci. 84:2327-2335.

Veerkamp, R. F., J. K. Oldenbroek, H. J. van der Gaast, and J. H. J. van der Werf. 2000. Genetic correlation between days until start of luteal activity and milk yield, energy balance and live weights. J. Dairy Sci. 83:577-583.

Wall, E., M. P. Coffey, and S. Brotherstone. 2007. The relationship between body energy traits and production and fitness traits in first-lactation dairy cows. J. Dairy Sci. 90:1527-1537.

Walsh, R. B., J. S. Walton, D. F. Kelton, S. J. LeBlanc, K. E. Leslie, and T. F. Duffield. 2007. The effect of subclinical ketosis in early lactation on reproductive performance of postpartum dairy cows. J. Dairy Sci. 90:2788-2796.

Wathes, D. C., N. Bourne, Z. Cheng, G. E. Mann, V. J. Taylor, and M. P. Coffey. 2007. Multiple correlation analyses of metabolic and endocrine profiles with fertility in primiparous and multiparous cows. J. Dairy Sci. 90:1310-1325.

Westwood, C. T., I. J. Lean, and J. K. Garvin. 2002. Factors influencing fertility of Holstein dairy cows: A multivariate analysis. J. Dairy Sci. 85:3225-3237.

Whitaker, D. A. 2004. Metabolic profiles. Pages 804-819 in Bovine Medicine, Diseases and Husbandry of Cattle. 2nd ed. A. H Andrews with R. W. Blowey, H. Boyd, and R. G. Eddy, ed Blackwell Publishing, Oxford, UK. 